\title{
Cuidados Intensivos para Pacientes em Pós-Operatório de Cirurgia Bariátrica*
}

\section{Intensive Care of Postoperative Patients in Bariatric Surgery}

\author{
Giselle Domingues Sanches', Fernanda M. Gazoni', Renata Koda Konishi', \\ Hélio Penna Guimarães ${ }^{2,3,4}$, Letícia Sandre Vendrame ${ }^{2,3}$, Renato Delascio Lopes ${ }^{5}$
}

\section{RESUMO}

JUSTIFICATIVA E OBJETIVOS: A obesidade é considerada uma epidemia e afeta mais de 300 milhões de pessoas no mundo. A sua prevalência vem aumentando significativamente nos últimos anos e a mortalidade do paciente obeso crítico, em alguns estudos, mostrase maior, especialmente em pacientes cirúrgicos que necessitam de UTI. Este estudo teve como objetivo ressaltar as particularidades das condutas em Medicina Intensiva no pós-operatório do paciente obeso submetido à cirurgia bariátrica.

CONTEÚDO: A taxa dos pacientes obesos na UTI é de $9 \%$ a $26 \%$ e o aumento no número de cirurgias bariátricas tem elevado o fluxo de obesos internados em UTI. A obesidade tem várias particularidades fisiopatológicas que devem ser conhecidas para melhor manuseio pós-operatório. Entre elas estão as restrições pulmonares, que leva ao aumento do número de com-

1. Médica Especializanda da Disciplina de Clínica Médica da UNIFESP-EPM

2. Médico Assistente da UTI da Disciplina de Clínica Médica da UNIFESP-EPM

3. Título de Especialista em Medicina Intensiva pela AMIB-AMB

4. Médico da Divisão de Pesquisa do Instituto Dante Pazzanese de Cardiologia.

5. Médico Assistente da UTI da Disciplina de Clínica Médica da UNIFESP-EPM.

*Recebido da UTI da Disciplina de Clínica Médica da Universidade Federal de São Paulo, Escola Paulista de Medicina (UNIFESP-EPM), São Paulo,SP.

Apresentado em 13 de fevereiro de 2007

Aceito para publicação em 05 de abril de 2007

Endereço para Correspondência:

Dra. Giselle Domingues Sanches

Rua Napoleão de Barros, 715, 3 Andar

UTI da Disciplina de Clínica Médica da UNIFESP-EPM

04024-002 São Paulo, SP

E-mail: gisellesanches@ig.com.br

(C)Associação de Medicina Intensiva Brasileira, 2007 plicações, o fator de risco para síndrome coronariana aguda eventos trombóticos.

CONCLUSÕES: O número de pacientes submetidos à cirurgia bariátrica está aumentando, o que leva a maior número de pacientes obesos internados na UTI. As alterações fisiológicas da obesidade, juntamente com as complicações de um paciente crítico, são desafios para a prática médica. Portanto, é relevante conhecer a fisiopatologia da obesidade, o tratamento rotineiro das complicações no pós-operatório de cirurgia bariátrica.

Unitermos: cirurgia bariátrica, cuidados pós-operatórios, Medicina Intensiva.

\section{SUMMARY}

BACKGROUND AND OBJECTIVES: Obesity is an epidemic disease reaching more than 300 million people all over the world. Its prevalence has increased during the past few years and according to some studies its mortality in the critically ill patient seems to be much higher, especially among patients who were submitted to surgery. This study has as objective to discuss some particularities of managing obese patients in the intensive care unit after bariatric surgery.

CONTENTS: The rate of obese patient in the ICU ranges from $9 \%$ to $26 \%$ and the increase in the number of bariatric surgeries has raised the number of obese patients in the ICU. It is important to know the physiopathology of obesity and to treat its particularities during the postoperative care. Such as pulmonary restriction, that causes an increase in pulmonary complications, coronary artery disease and thromboembolic events.

CONCLUSIONS: The number of patients that undergo to bariatric surgery has increased; therefore, the number of obese patients in the ICU has also risen. Different physiological events and complications in obese patients are challenges to clinical practice. The knowledge of obese physiopathology helps in the managing routine procedures and complications after bariatric surgery. 
Key Words: bariatric surgery, intensive care medicine, postoperative care

\section{INTRODUÇÃO}

A obesidade é considerada uma epidemia e afeta mais de 300 milhões de pessoas em todo o mundo. Nos Estados Unidos, $60 \%$ dos homens e $51 \%$ das mulheres têm sobrepeso ou obesidade, sendo $30 \%$ com IMC (índice massa corpórea), ou seja: peso $(\mathrm{kg}) /$ altura $^{2}\left(\mathrm{~m}^{2}\right)$ maior que 30 e $5 \%$ mórbidos (IMC > 40) ${ }^{1}$. No Brasil, $40 \%$ da população está acima do peso e cerca de $10 \%$ dos gastos de saúde pública estão relacionados à obesidade $^{2}$.

Significativos avanços têm sido alcançados no tratamento farmacológico da obesidade mórbida, no entanto, a cirurgia bariátrica é considerada mais eficaz para esses pacientes e é capaz de resolver boa parte das comorbidades causadas pela obesidade ${ }^{3}$. Os critérios estabelecidos para indicação de cirurgia bariátrica são: IMC > $40 \mathrm{~kg} / \mathrm{m}^{2}$ ou com IMC $\geq$ $35 \mathrm{~kg} / \mathrm{m}^{2}$ quando associado a outras comorbidades. Os pacientes que se enquadram nos critérios estabelecidos descritos, para ter confirmada a indicação da cirurgia, deverão ainda não possuir doenças endócrinas específicas; transtorno mental (alcoolismo, drogadição); ou condições físico-clínicas que contra-indiquem cirurgias de grande porte (cirrose, cardiopatia, pneumopatia, insuficiência renal crônica e outras) $)^{4,5}$.

Quatro opções de técnica cirúrgica são atualmente empregadas: bypass gástrico com $Y$ de Roux, bandagem gástrica vertical, bandagem gástrica ajustável por via laparoscópica e switch duodenal. Comparativamente, a mortalidade é de $0,5 \%$ a $1 \%$ para as cirurgias abertas versus $0,1 \%$ a $0,5 \%$ para as cirurgias laparoscópicas, respectivamente. A morbidade para ambas as modalidades cirúrgicas foi semelhante, em torno de $5 \%$. Apenas $6 \%$ a $24 \%$ dos pacientes submetidos à cirurgia bariátrica necessitam mais de $24 \mathrm{~h}$ de internação em UTI, número maior em cirurgias "abertas" (21\%) quando comparado com a laparoscópica $(7,6 \%)^{1}$.

O número de pacientes obesos internados em UTI é cerca $9 \%$ a $26 \%$, sendo $1,4 \%$ a $7 \%$ obesos mórbidos. Devido ao aumento na indicação de cirurgia bariátrica, tornou-se um desafio oferecer suporte intensivo que esses pacientes necessitam. Neste cenário, conhecer as particularidades fisiopatológicas desses pacientes torna-se condição indispensável para o seu manuseio clínico ${ }^{4,5}$.

\section{FISIOPATOLOGIA DA OBESIDADE}

A obesidade é caracterizada por estado inflamatório crônico que diminui tanto a capacidade imunológica quanto metabólica, além de cursar com hipercoagulabilidade e resistência à insulina ${ }^{1}$. O tecido adiposo libera fator de necrose tumoral (TNF) alfa e interleucina 6 (IL-6). Há também alteração na mobilidade e ativação dos neutrófilos; na concentração do fibrinogênio e do inibidor do ativador do plasminogênio 1, diminuição da antitrombina III e fibrinólise ${ }^{6}$. A resistência insulínica gera hiperinsulinemia, aumenta a retenção renal de sódio, ativação do sistema nervoso simpático e aumento da reatividade vascular. Também gera dislipidemia, hiperuricemia e intolerância à glicose ${ }^{1}$.

$\mathrm{O}$ aumento do IMC exige aumento no trabalho vascular, respiratório e metabólico, resultando em importante redução da reserva fisiológica (Figura 1).

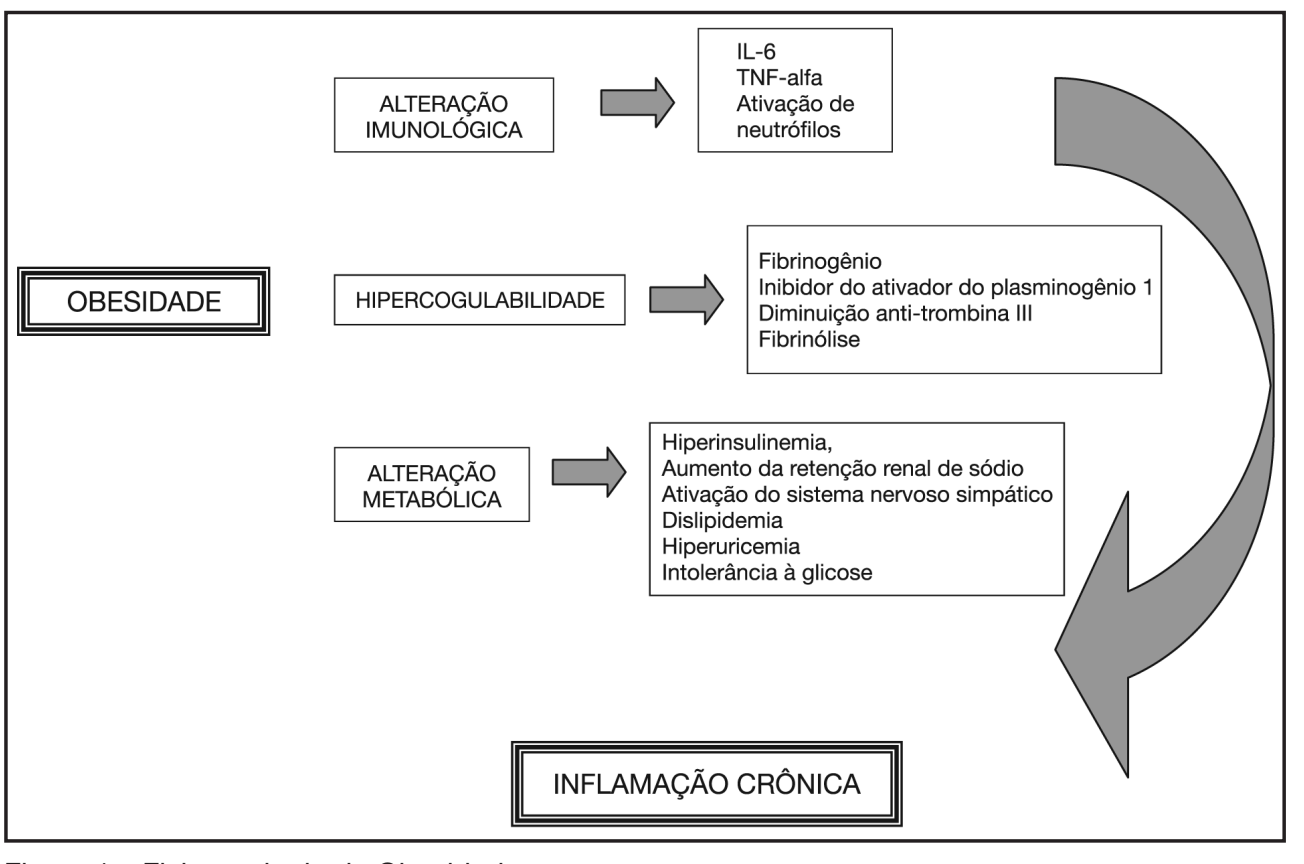

Figura 1 - Fisiopatologia da Obesidade

Figura 1-Fisiopatologia da Obesidade 


\section{FARMACOLOGIA}

Os pacientes obesos, sem insuficiência renal, têm taxa de filtração glomerular alta, o que aumenta a depuração de fármacos com excreção renal. Devido aos vários fatores associados à obesidade que também predispõem à falência renal, o cálculo da depuração de creatinina pela urina se torna importante. O aumento do tecido adiposo altera o volume de distribuição dos fármacos lipofílicos (p. ex.: propofol). O acúmulo desses fármacos no tecido adiposo faz com que se aumente a dose necessária para atingir o mesmo efeito e prolonga sua meia-vida, diminuindo ou retardando a sua eliminação. A dose necessária desses medicamentos é mais bem calculada pelo peso corporal atual (PCA) que pelo corporal ideal (PCl).

Ao contrário dos fármacos lipofílicos, os hidrofílicos ( $p$. ex: fentanil) penetram fracamente no tecido adiposo e por isso estão mais bem relacionados com o peso ideal. O uso do cálculo da dose pelo peso atual pode levar a intoxicação. Um recurso também freqüentemente utilizado em Medicina Intensiva e Anestesiologia é o uso do peso corrigido ( $\mathrm{PC}$ ) que consiste da soma de $40 \%$ do excedente do peso ao peso ideal (altura-100) do paciente (Tabela 1).

Tabela 1 - Cálculo da Dose Corrigida para Pacientes Obesos Mórbidos ${ }^{1}$.

\begin{tabular}{lc}
\hline Fármacos & Dose Corrigida pelo Peso \\
\hline Propofol & $\mathrm{PCA}$ \\
$\begin{array}{l}\text { Benzodiazepínico } \\
\quad \text { Dose única } \\
\quad \text { Infusão contínua }\end{array}$ & $\mathrm{PCA}$ \\
Fentanil & $\mathrm{PCl}$ \\
Vancomicina & $52 /\left(1+\left[196.4 \times \mathrm{x}^{-0.025 \mathrm{PCA}-53.66] / 100)}\right.\right.$ \\
Aminoglicosídeo & $\mathrm{PCA}$ \\
Fluoroquinolona & $\mathrm{PCl}+(40 \times[\mathrm{PCA}-\mathrm{PCl})]$ \\
Drotrecogina alfa ativada & $\mathrm{PCl}+(40 \times[\mathrm{PCA}-\mathrm{PCl})]$ \\
\hline
\end{tabular}

\section{SISTEMA RESPIRATÓRIO E VENTILAÇÃO MECÂNICA}

O paciente obeso geralmente apresenta doença pulmonar restritiva devido ao maior volume de sangue pulmonar e espessura da parede torácica. Além disso, o diafragma encontra-se em posição anormal, há resistências nas vias aéreas superiores e aumento na produção de $\mathrm{CO}_{2}$. Nessa condição, este é um paciente que pode estar associado a padrão obstrutivo, com diminuição na capacidade vital e no volume-minuto ${ }^{7}$.

A intubação traqueal pode estar dificultada por alteração na anatomia cervical, macroglossia e orofaringe redundante; há também maior risco de broncoaspiração no paciente obeso, particularmente quando houver hérnia hiatal, aumento da pressão intra-abdominal, acidez gástrica e maior volume gástrico ${ }^{7}$.

A ventilação mecânica é necessária em $0,6 \%$ a $7,3 \%{ }^{6}$ dos pacientes no pós-operatório de cirurgia bariátrica. O volume-corrente deve ser calculado pelo peso ideal, diminuindo o risco de barotrauma, distensão alveolar e alta pressão nas vias aéreas ${ }^{8}$. A posição de Trendelemburg invertida a $45^{\circ}$ parece ser melhor que a supina ${ }^{7}$, pois há aumento da pressão arterial de oxigênio $\left(\mathrm{PaO}_{2}\right)$, do volume-corrente (VC) e redução na freqüência respiratória.

A restrição pulmonar do pós-operatório persiste por até duas semanas, o que aumenta o número de complicações pulmonares, como retenção $\mathrm{CO}_{2}$, atelectasia e infiltrado broncopulmonar ${ }^{1}$. A fisioterapia respiratória, por pressão positiva intermitente (CPAP), tem efeito benéfico para resolução destas complicações ventilatórias comuns no pós-operatório. O CPAP restaura a capacidade funcional residual, aumenta oxigenação, melhora a potência muscular, porém esse efeito não é sustentado. O uso do CPAP não aumenta o risco de deiscência de sutura, apesar de causar distensão gástrica ${ }^{9}$.

\section{CARDIOVASCULAR}

A obesidade é fator de risco independente para síndrome coronariana aguda, diabete melito e hipertensão. A obesidade abdominal é importante fator de risco para o infarto agudo do miocárdio (OR 2,24, IC 2,06-2,45, p $<0,0001)^{10,11}$. Cerca de $60 \%$ dos obesos apresentam hipertensão arterial sistêmica. A maioria é sedentária e por isso oligossintomática para angina.

O sobrepeso promove pré e pós-carga elevadas, pois há aumento da volemia, níveis de renina, aldosterona, mineralocorticóide e catecolaminas. Pode haver hipercinesia, hipertrofia miocárdica e disfunção diastólica, resultando em aumento do trabalho cardíaco ${ }^{1}$. Medidas de pressão arterial não-invasivas não são fidedignas e, por isso, quando há suspeita de instabilidade hemodinâmica está indicada a monitorização com cateter arterial.

\section{NUTRIÇÃO}

A cirurgia bariátrica pode ser dividida em dois grupos: restritivas e malabsortivas com ou sem restrição. As necessidades nutricionais variam dependendo do grau de restrição e de malabsorção causada 
pela cirurgia e também da área do intestino usado para o bypass ${ }^{12}$.

O procedimento restritivo, como bandagem laparoscópica ajustável, tem como objetivo a perda de peso por restrição do volume total de alimento ingerido ${ }^{12}$.

Dos procedimentos malabsortivos, o mais utilizado é o bypass gástrico em $Y$ de Roux que envolve o fundo gástrico, corpo e antro, duodeno e jejuno. Conseqüentemente, há maior risco de se desenvolver deficiência de ferro, vitamina B12, folato e cálcio. Isso porque é no estômago que o ácido converte o ferro da forma ferrosa em férrica e é no duodeno onde ocorre a sua absorção. A absorção da vitamina B12 fica muito prejudicada, pois há impossibilidade da ligação com fator intrínseco condicionado à integrada do duodeno ${ }^{13,14}$. A deficiência de folato não é muito comum, mas pode ocorrer quando há baixa ingestão associada.

\section{Modificações da Dieta}

A dieta se inicia com líquidos. Deve-se estar atento para a síndrome de Dumping quando o piloro é afetado ou quando houver bypass do duodeno. Uma a duas semanas após a cirurgia pode-se iniciar alimentos pastosos e após um mês, dieta leve. As recomendações variam de um profissional para o outro, assim como a tolerância alimentar depende de cada paciente. Náuseas e vômitos são geralmente causadas por superalimentação ou pela deglutição de fragmentos maiores de alimento. A desidratação é muito comum após o procedimento cirúrgico e é atribuída principalmente à baixa ingestão de líquidos. Vômito e diarréia podem exacerbar a desidratação. Como os pacientes não podem ingerir grande quantidade de líquidos, deve-se estimular a ingestão de pequenas quantidades com maior freqüência ${ }^{15}$.

O paciente obeso deve ter a nutrição parenteral total (NPT) iniciada 3 a 5 dias após a cirurgia, se estiver impossibilitado de dieta oral. Deve-se tomar cuidado com a síndrome de realimentação; portanto, inicia-se com $50 \%$ da necessidade e do volume estimado nas primeiras $24 \mathrm{~h}$.

Devem-se monitorar os valores de potássio, magnésio e fósforo a cada 3 dias e calcular uma solução hipocalórica e normo-protéica. A quantidade de calorias a ser fornecida deve ser de 15 a 20 por quilo de peso corporal ajustado (peso atual - peso ideal x $25 \%+$ peso ideal). A proteína deve ser de 1 a $2 \mathrm{~g}$ por quilo de peso ajustado. Gastrostomia pode ser considerada no pósoperatório crítico prolongado ${ }^{16}$.

\section{Síndrome de Dumping}

É uma resposta fisiológica aos açúcares simples que se caracteriza por tremor, sudorese, sensação de mal estar, taquicardia e, muitas vezes, intensa diarréia. Isso decorre do poder osmótico dos açúcares simples além da liberação de insulina ${ }^{16}$.

\section{TROMBOSE}

A obesidade é um fator de risco para eventos trombóticos, sendo que o IMC $>40 \mathrm{~kg} / \mathrm{m}^{2}$ é fator de risco independente para morte súbita por tromboembolismo pulmonar (TEP) agudo no pós-operatório $(p<0,001)^{17}$. A mortalidade total chega a $2 \%$ e tem como causa principal o TEP ${ }^{17,18}$.

A trombose venosa profunda (TVP) e o TEP ocorrem em cerca de $2 \%$ a $4 \%$ de pacientes submetidos a bypass gástrico em $Y$ de Roux ${ }^{18-20}$, com mortalidade de $25 \%$ a $67 \%$ em pacientes que desenvolvem embolia pulmonar $^{17,18,21}$.

Gonzáles e col. ${ }^{22}$, em estudo prospectivo realizado com 660 pacientes, a profilaxia pode ser realizada com compressão mecânica intermitente no intra-operatório e com heparina de baixo peso molecular de acordo com o IMC. Pacientes com IMC $<50 \mathrm{~kg} / \mathrm{m}^{2}$ receberam $40 \mathrm{mg}$, por via subcutânea de enoxaparina, com IMC $\geq 50 \mathrm{~kg} / \mathrm{m}^{2}, 30 \mathrm{mg}$ duas vezes ao dia e IMC $\geq 60 \mathrm{~kg} / \mathrm{m}^{2}$ ou com imobilidade relativa receberam profilaxia por período maior, de 10 a 14 dias $^{22}$. O uso de heparina não-fracionada, heparina de baixo peso molecular ou de compressão pneumática intermitente dos membros inferiores, está recomendado para pacientes de alto risco, que serão submetidos à cirurgia eletiva abdominal, com início 1 a 2 horas antes do procedimento ${ }^{23}$.

Os pacientes em alto risco, com obesidade central, estase venosa e antecedente de TEP podem se beneficiar com a implantação de filtro de veia cava ${ }^{22,24}$. O evento tromboembólico ocorre em média cinco dias após a cirurgia, variando de 0 a 21 dias $^{22}$.

\section{ACESSO VASCULAR}

A anatomia distorcida dificulta o acesso venoso central. Verificou-se menor número de complicações com a utilização da punção da veia jugular interna. Deve haver maior vigilância contra infecção. O cuidado com áreas intertriginosas precisa ser maior, pois o tecido é macerado e são áreas de difícil higienização'. 


\section{COMPLICAÇÕES}

\section{Deiscência de Sutura}

Essa complicação é rara, mas pode ser fatal se não for diagnostica e corrigida rapidamente. Os sinais mais comuns são taquicardia, insuficiência respiratória (saturação de oxigênio $<92 \%$, freqüência respiratória $>24)$, dor abdominal e derrame pleural à esquerda isolado. $\mathrm{O}$ principal diagnóstico diferencial é o tromboembolismo venoso pulmonar. O tratamento deve ser laparotomia exploradora de urgência1.

\section{Rabdomiólise e Insuficiência Renal}

A rabdomiólise induzida por pressão é complicação rara no pós-operatório que resulta da compressão prolongada dos músculos durante a cirurgia, principalmente os das regiões lombares, dos membros inferiores e glúteos. A prevenção se faz com o posicionamento da mesa cirúrgica, redução do tempo operatório e avaliação criteriosa durante o pós-operatório. Diabete e alto IMC são os maiores fatores de risco. Os sintomas são dor e dormência muscular, lesão de pele, urina acastanhada, creatinoquinase $>5000 \mathrm{UI}$. O tratamento é feito com hidratação vigorosa e manitol. As complicações são síndrome compartimental, insuficiência renal crônica e óbito'.

A prevalência de insuficiência renal aguda é de $50 \%$ no pós-operatório de cirurgia bariátrica. Hipovolemia, oclusão tubular, acidose e aumento de radicais livres são as principais causas. Os fatores preditivos de IRA na rabdomiólise são idade avançada, CK > 16000 UI, hipoalbuminemia e sepse. Geralmente a função renal volta ao normal, porém vale lembrar que a hemofiltração facilita rápida remoção da mioglobina, diminuindo as lesões glomerulares ${ }^{25}$.

\section{CONCLUSÃO}

Devido ao aumento no número de cirurgias bariátricas o fluxo de pacientes obesos em UTI aumentou ${ }^{3}$ e tornou-se um desafio na prática clínica por apresentarem diversas alterações fisiológicas ${ }^{1}$. Conhecer essas particularidades contribui para o seu manuseio, mas novos estudos ainda são necessários para contribuir na abordagem de pacientes obesos cirúrgicos.

\section{REFERÊNCIAS}

01. Pieracci FM, Barie PS, Pomp A - Critical care of the bariatric patient. Crit Care Med, 2006;34:1796-1804.

02. FUNDAÇÃO IBGE. Pesquisa de Orçamentos Familiares - POF 2002 2003,Brasília, Dez 2004. disponível em http://: www. ibge. gov.br / home /presidencia/ noticias. Acessado em 20/10/2006

03. Buchwald $\mathrm{H}$ - Consensus Conference Statement: Bariatric surgery for morbid obesity: health implications for patients, health professionals, and third-party payers. J Am Coll Surg, 2005;200:593-604.

04. Poulose BK, Griffin MR, Zhu Y et al - National analysis of adverse patient safety events in bariatric surgery. Am Surg, 2005;71:406-413.

05. El-Solh AA - Clinical approach to the critically ill, morbidly obese patient. Am J Respir Crit Care Med, 2004;169:557-561.

06. Wu EC, Barba CA - Current practices in the prophylaxis of venous thromboembolism in bariatric surgery. Obes Surg, 2000;10:7-14.

07. Burns SM, Egloff MB, Ryan B et al - Effect of body position on spontaneous respiratory rate and tidal volume in patients with obesity, abdominal distention and ascites. Am J Crit Care, 1994;3:102-106.

08. Helling TS, Willoughby TL, Maxfield DM et al - Determinants of the need for intensive care and prolonged mechanical ventilation in patients undergoing bariatric surgery. Obes Surg, 2004;14:1036-1041.

09. El-Solh A, Sikka P, Bozkanat E et al - Morbid obesity in the medical ICU. Chest, 2001;120:1989-1997.

10. Yusuf S, Hawken S, Ounpuu $S$ et al - Effect of potentially modifiable risk factors associated with myocardial infarction in 52 countries (the INTERHEART study): case-control study. Lancet, 2004;364:937-952.

11. Piegas LS, Avezum A, Pereira JC et al - Risk factors for myocardial infarction in Brazil. Am Heart J, 2003;146:331-338.

12. Rationale for the surgical treatment of morbid obesity. Disponível em http://www.asbs.org. acessado em 20/10/2006

13. MacLean LD, Rhode BM, Shizgal HM - Nutrition following gastric operations for morbid obesity. Ann Surg, 1983;198:347-355.

14. Halverson JD - Micronutrient deficiencies after gastric bypass for morbid obesity. Am Surg, 1986;52:594-598.

15. Elliot K - Nutritional considerations after bariatric surgery. Crit Care Nurs Q, 2003;26:133-138.

16. Kushner R - Managing the obese patient after bariatric surgery: a case report of severe malnutrition and review of the literature. J Parenter Enteral Nutr, 2000;24:126-132.

17. Blaszyk H, Bjornsson $\mathrm{J}$ - Factor $\mathrm{V}$ leiden and morbid obesity in fatal postoperative pulmonary embolism. Arch Surg, 2000;135:1410-1413.

18. Stoopen-Margain E, Fajardo R, Espana N et al - Laparoscopic Roux-en$Y$ gastric bypass for morbid obesity: results of our learning curve in 100 consecutive patients. Obes Surg, 2004;14:201-205.

19. Schauer PR, Ikramuddin S, Gourash W et al - Outcomes after laparoscopic Roux-en-Y gastric bypass for morbid obesity. Ann Surg, 2000;232:515-529.

20. DeMaria EJ, Sugerman HJ, Kellum JM et al - Results of 281 consecutive total laparoscopic Roux-en-Y gastric bypasses to treat morbid obesity. Ann Surg, 2002;235:640-647.

21. Shepherd MF, Rosborough TK, Schwartz ML - Heparin thromboprophylaxis in gastric bypass surgery. Obes Surg, 2003;13:249-253.

22. Gonzalez R, Haines K, Nelson LG et al - Predictive factors of thromboembolic events in patients undergoing Roux-en-Y gastric bypass. Surg Obes Relat Dis, 2006;2:30-35

23. Sapala JA, Wood MH, Schuhknecht MP et al - Fatal pulmonary embolism after bariatric operations for morbid obesity: a 24-year retrospective analysis. Obes Surg, 2003;13:819-825.

24. Nicolaides AN - Prevention of venous thromboembolism. J Vasc Br, 2002;1:133-170.

25. Snider RD, Kruse JA, Bander JJ et al - Accuracy of estimated creatinine clearance in obese patients with stable renal function in the intensive care unit. Pharmacotherapy, 1995;15:747-753. 